\title{
TRAINING STAND FOR SIMULATION OF HYBRID ENERGY SYSTEM AND GSM REMOTE CONTROL
}

\author{
Alexey Lavrov, Anatolii Lupin \\ Peter the Great Saint-Petersburg Polytechnic University, Russia \\ alecse-lavro@rambler.ru, avlu@rambler.ru
}

\begin{abstract}
SMS and GPRS-based remote control systems are widespread currently. They are applicable to various objects. When studying such systems, students need to master a number of special skills, namely, software development for contemporary GSM modems (e.g., Neoway M660), which are necessary to exchange information and to learn how to develop user graphic interfaces (GUI) central control panel (CCP) engaged in information processing and management. As a sample of remote control system an autonomic Hybrid Energy System (HES) for GSM base transceiver station is considered. Remote control for HES is important, because the efficiency of such control allows increasing the HES reliability, which influences largely the entire reliability of cellular systems. Meanwhile, it is almost impossible to deploy real HES in laboratory conditions. This report proposes an approach, where software developments for control algorithm tests make use of HES logical mathematical model (simulator). The HES model includes a communication controller emulating the properties of a real HES to some extent. The first option assumes a computer running a LabVIEW model of HES with communication controller, which is connected to a GSM modem via the serial interface and another external USB GSM modem connected to the same computer. The CCP GUI is running in the LabVIEW environment locally. This approach leads to the possibility of not only delivering theoretical knowledge, but making students, firstly, to acquire practical skills to operate GSM-modules with AT-commands, secondly, to use GSM protocols and, thirdly, to implement software dispatching consoles. Applying this method caused academic performance increase from 3.9 up to 4.3. Moreover, students have got a positive motivation to proceed with remote control system development.
\end{abstract}

Keywords: Hybrid Energy System (HES), GSM remote control, training stand.

\section{Introduction}

HES is designed to provide a stable power supply of residential or industrial facility. As an alternative to electricity, solar, wind, fuel and accumulated reserve battery are used. This paper considers GSM Base Circular Stations (BCS). BCS location requires a possibility of remote control. Remote control of BCS HES via GSM channels of central control panel (CCP) is shown in Fig. 1.

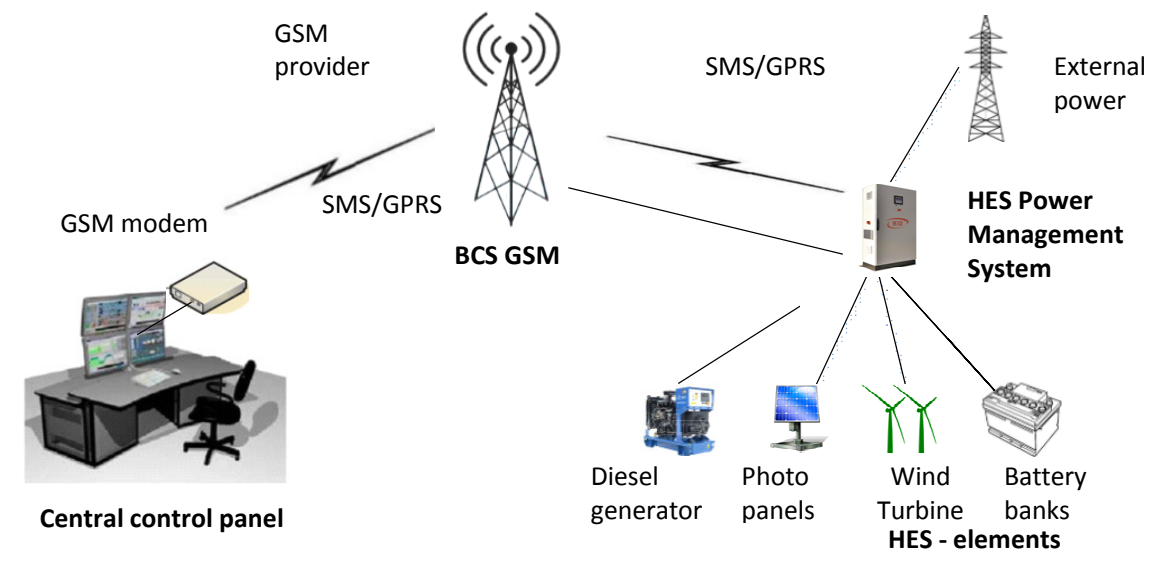

Fig.1. HES GSM remote control

Typical HES includes the following blocks: wind turbine, photo voltaic panels, battery banks (BB), diesel systems (diesel generator), external power energy. At the moment HES is developed, e.g., by R.I.D. GmbH (Germany) [1], Genelec (France), Kubota (Japan), Master (Italy) [2], Electrosystems (Russia) [3] and others.

The versatility of the complex HES and CCP lies in its ability to:

- serve both main and backup power supply;

- use the alternative sources of electricity like diesel generator units or external power energy network; 
- adapt the composition and configuration of the specific conditions and requirements;

- work in offline mode while unattended within a specified time;

- work reliably under temperatures from -40 to +50 degrees Celsius;

- provide the remote monitoring and control of operating modes of the complex via standard communication channels or via the Internet.

Remote management of HES is topical; the promptness of such control allows to increase the reliability of HES, on which reliability of operation and the systems of cellular communication largely depends.

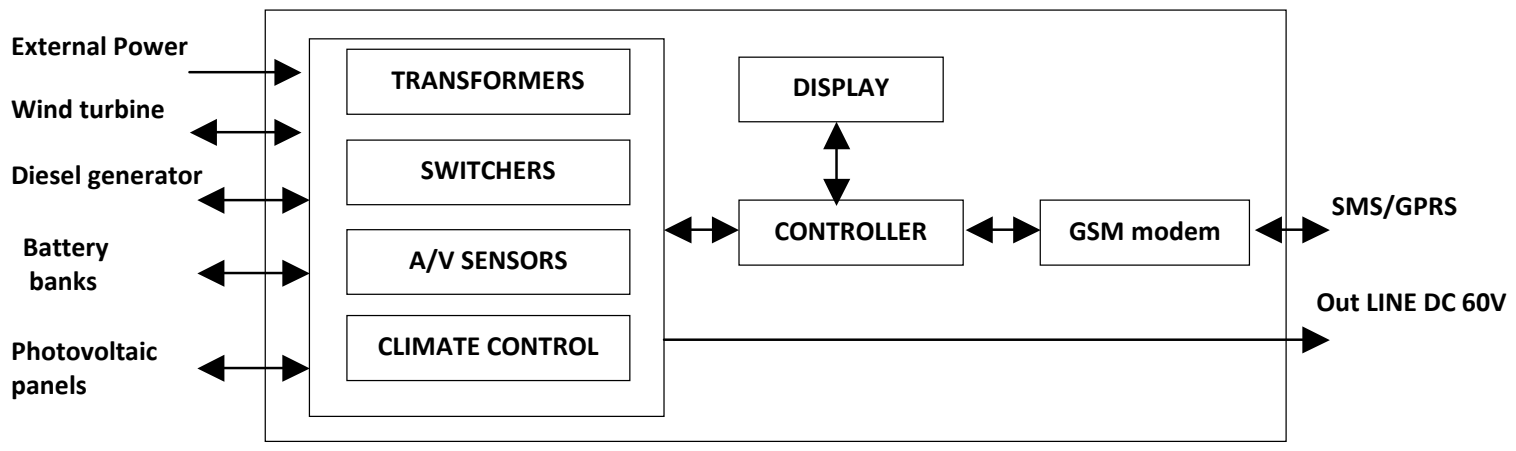

Fig. 2. Block-diagram HES Power Management System

HES includes the Power Management System (Fig. 2). The prototype device is RID BMC-908 [1]. PMS contains transformers with detectors, switchers, A/V sensors, climate control. The centerpiece of the system is the controller, which manages all the PMS elements and connects the output power line. In turn, the controller is operated from the CCP via SMS or GPRS.

A lot of research on HES concerns the choice (and optimization) of a number of HES items based on mathematical models [4-8]. The results are presented in reports at conferences [5;6] and scientific works of the authors [7; 8]. HES control algorithms are outlined in [4-8].

For teaching data transfer protocols GSM offers several educational booths. One of them [9] is a training stand "GSM cellular system". It contains a device for GSM controller programming and learning modes and ways of sending SMS-messages. Another option-educational complex wireless data transmission for GPS/GSM DGS-200 [10] production company K\&H Mfg. co., Ltd. DGS-200 covers two topics in modern communication technology: GPS and GSM/GPRS technology.

The authors of the report propose an alternative approach to creating an educational facility for remote control simulation HES partially outlined in [11].

\section{Materials and methods}

The basic concept of building a training facility for students of the Faculty of Computer Systems and Software Technology "is that based on a relatively simple and affordable hardware able to develop modern technologies, to develop graphical interfaces, create models of individual systems and remotely manage based on wireless communications in real time".

The composition of the stand includes the PC with the characteristics necessary for the installation and operation of Windows OS 7 (or higher), debug Board M660-DK-MOD [12] with antenna ESGGSM-02, GSM modem and provider. For the connection of equipment by PC one needs 2 free USB ports. Board M660-DK-MOD contains: module Neoway M660, board M5X0-PWR, providing power and communications module via USB interface with PC, and SMA antenna connector. A SIM Card is to be added.

The main characteristics of the module Neoway M660 are listed below.

- Frequency range 850/900/1800/1900 MHz.

- Data transfer protocols:

- GPRS class 12, maximum speed $85.6 \mathrm{Kbps}$;

- SMS: reception/transmission, point-to-point MO/MT, broadcast mode; 
- Built-in TCP/UDP protocol stack/FTP/DNS.

- AT command set: GSM 07.05, 07.07, proprietary extensions.

- Microprocessor: ARM7-EJ @ 104MHz, 32Mbits SRAM, 32 64Mbits NOR Flash.

The stand includes Windows OS 7 (or higher) and LabVIEW one of the versions [13]. As a terminal program (AT) used SecureCRT 7.3, you can also use the branded software offered by Neoway Technology-Neo CMUX Tool [14].

Methodical provision of the stand is the concept of the separation of the common model HES and CCP on two levels. The lower level is the logic-mathematical HES-model and the Simulator becomes as further signals and events. The upper level includes the CCP-model and interaction of HES with CCP. At the booth of the bottom tier the PC and module M660-DK-MOD are implemented, and the upper level, the same PC with modem GSM.

\section{Results and discussion}

General principles of organization of remote management of HES convenient study are based on a model that describes a very simple, but nevertheless frequently used structure of the HES, which includes the following main devices: a step-down transformer with a detector connected to the external power energy line, a diesel generator (DG), battery banks (BB), various sensors, switching device, shared Controller. If there is voltage in the external power energy line, HES produces a DC, 60 volts (nominal), which comes to the consumer from the output of the PMS.

With decreasing the external line voltage below the specified level or power, the power of the HES consumer will be from BB. Switching exercises the PMS Controller, which receives data from the sensors voltage. If electricity breaks for longer time (tens of minutes-hours) and the BB voltage is reduced to the minimum possible level, automatic starts DG, which can carry electricity within a time interval, defined by fuel reserve. During this interval, if necessary, taking into account statistical data on electricity failures in external line, on decision of the Operator CCP located in the Center, Emergency Brigade delivers the needed fuel. Charge or recharge of BB is from the external line or DG and operated by the BB controller that performs, as a general rule, other features: calculates the voltage levels and charge individual items and entire BB, produces training cycles and carries out exchange of information on the serial interface with the General controller. With a short-term loss of voltage in the transmission line (a few tens of minutes), sufficient energy stored in the BB is provided to power the consumer of the HES.

In the first phase of the model development the module M660 is operated. After you successfully configure the GPRS module, you must configure a TCP connection and connect to the server, and then you can start sharing with the CCP-model. Configuration is performed in two stages: configuring PPP (point-to-point protocol), then configure the TCP connection. Students work with the module M660 The first program (project) simulates the HES components, allows you to specify options for the main devices of HES and send data via the modem. To set the value of each parameter for each device software module is designed that implements the user interface window. The project was implemented in LabVIEW in the manner described in [15]. Data update time is several seconds and may be regulated within the framework of the technical capabilities of the system. It requires special knowledge and takes time. Details are not considered here.

Fig. 3 shows the HES virtual panel in the current mode of operation. Example energy comes from DG. All system settings are displayed, they are easy to read and understand. All of the windows in the title add the indicator (c), it means the option or permit obtained from the CCP.

In this case, climate control (1) works in a mode number 2 intensity of cooling, providing current temperature decrease ( 34 degrees $C$ ) to the required level ( 15 degrees).

External power voltage level (2) $165 \mathrm{~V}$ is below the required level, what triggered the alarm condition as signals RED LED.

BB also is not active (3) and is in an abnormal state (RED LED), in this case dilapidated due to the current charge of the battery, which is substantially below the alarm level, the charge mode is set to OFF (in this example), which implies that BB is not charging from the DG currently active. 


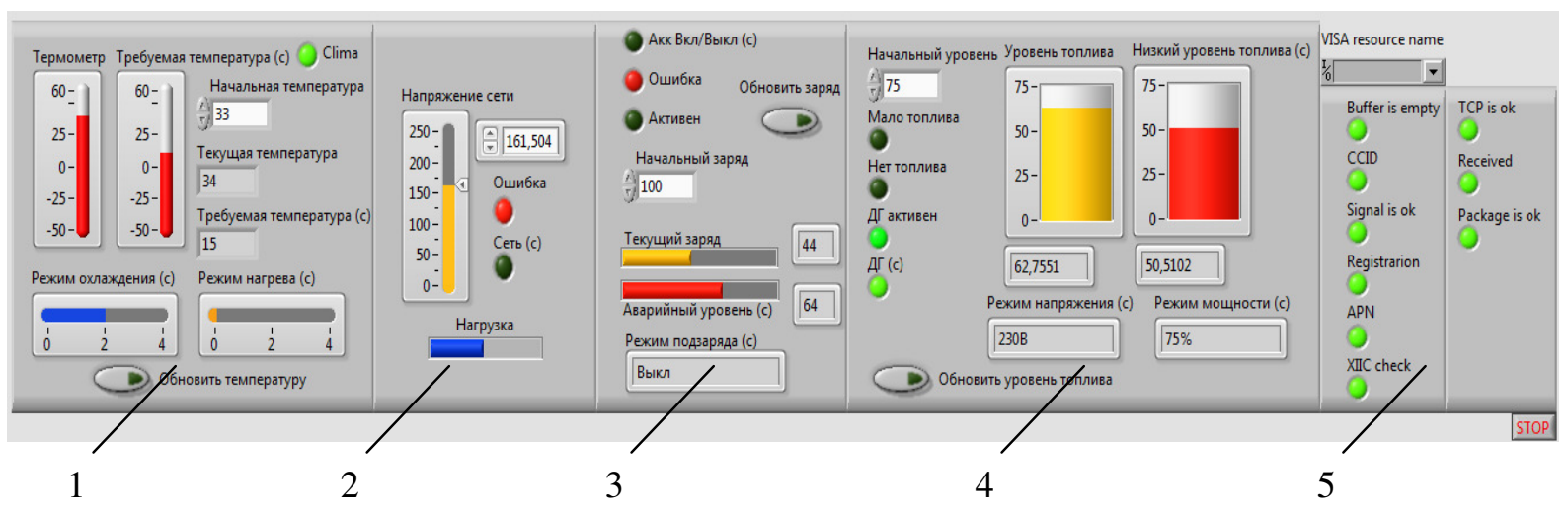

Fig.3. HES-model virtual panel

DG works in normal mode (4), as evidenced by the GREEN LED activity, it also shows that this way of supply is also allowed on the server side (CCP), as evidenced by the corresponding GREEN LED diode (c). Fuel level DG (YELLOW) is located above the lower limit value of the fuel level threshold (RED), for this reason, warning and emergency signals are not received and the corresponding diodes are no longer active. Rated voltage $230 \mathrm{~V}$ (followed by conversion to DC 60V nominal).

In the right part of the virtual panel (5) the unit is responsible for indication of successful connection to the TCP server and activity of this compound.

Second model (CCP model) - a program that supports the work of the CCP, forms requests by sending SMS to HES modem. For these requests, the program simulates HES-generated responses. To visualize the content of the responses the designed software module implements a set of window user interfaces. Virtual panel (Fig. 4) contains blocks of the general condition of systems (1) and monitoring blocks (2) and (3). Blocks of the general condition systems include: control unit BB, DG, control unit of network management and climatic setting. Health monitoring units, BB (2) and (3) of the DG'S status. This example shows permission presets and parameters BB, DG, external power (1), track current charge BB (2) and the current stock of fuel DG (3).

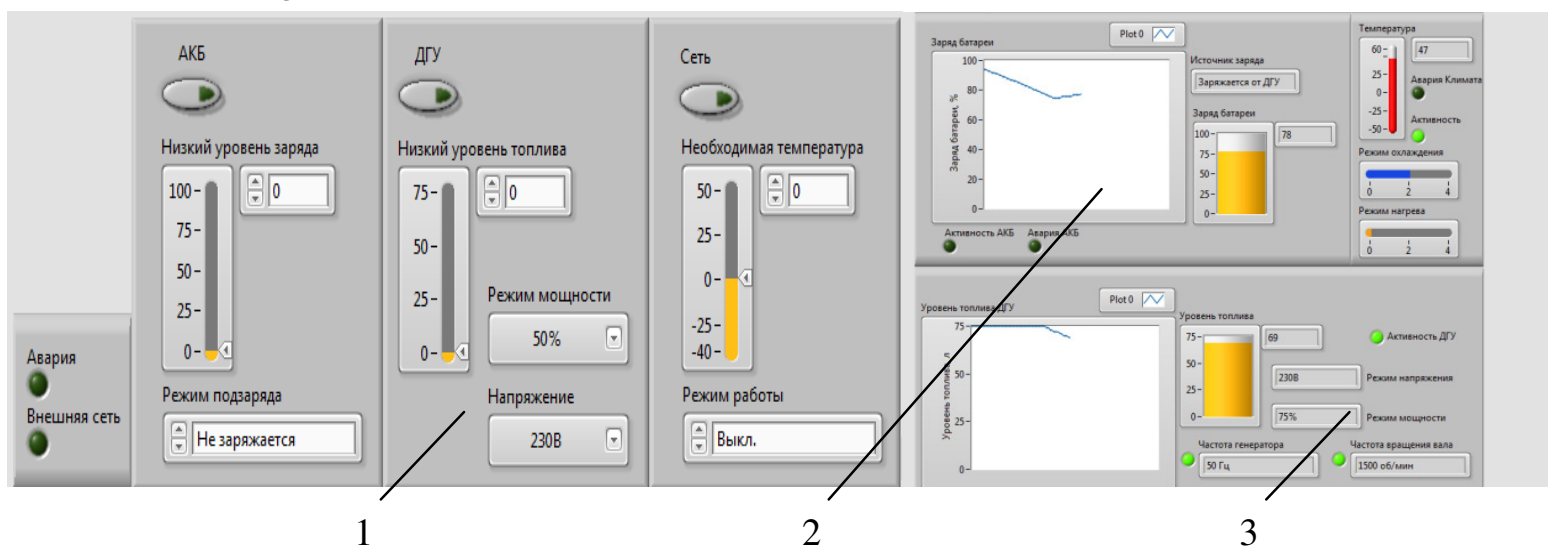

Fig.4. Virtual panel CCP-model: 1 - general condition and control; 2 -monitoring BB; 3 - monitoring DG

The HES model of GSM remote control implemented is used in the educational process, see "Remote management" course "External interfaces of the computer". It can also be used at an early stage of development HES to debug individual pieces of software. Over the past three years, the students created a few dozen projects with data transfer via SMS and GPRS-traffic, different GUI, with varying degrees of detail, using LabVIEW, Java, Qt Creator.

In Table 1 the comparative exam results are given. Students received practical skills to work with AT-commands, GSM modules, learned to use these plug-ins supported protocols, learned to develop user interfaces, perform the functions of the control boards. This, in turn, resulted in the average score 
for the exam raised from 3.9 points to 4.3 points. Future professionals have revealed better motivation to work in the area of development of systems for remote management.

Table 1

Comparative analysis of student performance at the rate

\begin{tabular}{|c|c|c|}
\hline Score for the exam & $\begin{array}{c}\text { Number of } \\
\text { students in 2015 }\end{array}$ & $\begin{array}{c}\text { Number of } \\
\text { students in 2017 }\end{array}$ \\
\hline 5 & 4 & 6 \\
\hline 4 & 3 & 5 \\
\hline 3 & 5 & 2 \\
\hline Total students & 12 & 13 \\
\hline $\begin{array}{c}\text { Average score for } \\
\text { the exam }\end{array}$ & 3.90 & 4.30 \\
\hline
\end{tabular}

\section{Conclusions}

1. Hardware-software training stand modelling complex systems GSM remote control is suggested. The cost of providing it is very low compared to the price of the PC.

2. Using the proposed methodology has resulted in that in addition to the theoretical knowledge students received practical skills to work with at-commands, GSM modules, learned to use these plug-ins supported protocols, learned to develop user interfaces,

3. The HES model is a typical training element (example) for creating a simulator of a complex system.

4. The CCP model in conjunction with the used equipment has essentially finished the CCP and can be used in real world design with minimal changes.

5. Skills acquired by students in the process of building projects and visibility reporting in real time, resulted in the average score for the exam risen from 3.9 points to 4.3 points. Future professionals have appeared further motivated to work in the development of systems for remote management.

\section{References}

[1] R.I.D. GmbH [online] [15.03.2018]. Available at: http://www.rid-gmbh.de.

[2] ЗАО "МНПО “Энергоспецтехника" (ZAO "MNPO "Energospectehnika") [online] [15.03.2018]. Available at: http://spectech.ru/about/brands/. (In Russian).

[3] Универсальный комплекс электроснабжения с использованием альтернативных источников "ГАРАНТ" (The universal complex of power supply with use of alternative sources "GARANT") [online] [15.03.2018]. Available at: http://www.electrosystems.ru/garant.php. (In Russian).

[4] Adefarati T., Bansal R.C. The Impacts of PV-Wind-Diesel-Electric Storage Hybrid System on the Reliability of a Power System. Proceedings of The $8^{\text {th }}$ International Conference on Applied Energy - ICAE2016. Energy Procedia, v. 105, pp. 616-621.

[5] Yang S.Y., Sung C.K., Huang C.Y. Optimization of Power Management Strategy for Parallel AirFuel Hybrid System. Proceedings of The $8^{\text {th }}$ International Conference on Applied EnergyICAE2016. Energy Procedia, v. 105, pp. 530-536.

[6] Chen J., Garcia H.E. Economic optimization of operations for hybrid energy systems under variable markets. Idaho National Laboratory [online] [15.03.2018]. Available at: https://www.osti.gov/pages/servlets/purl/1357460.

[7] Okundamiya M.S. Modelling and optimization of a hybrid energy system for GSM transceiver station sites in emerging cities. PhD Thesis, "Benin University", 2015.

[8] Alsharif M.H., Kim J. Optimal solar power system for remote telecommunication base stations: a case study based on the characteristics of South Korea's solar radiation exposure [online] [15.03.2018]. Available at: www.mdpi.com/2071-1050/8/9/942/pdf.

[9] Training equipment "cellular GSM" [online] [15.03.2018]. Available at: http://labstand.ru/expert/seti_evm/laboratoriya-seti-evm-standart.

[10] Training equipment DGS-200 GSM/GPS Experimental Set [online] [15.03.2018]. Available at: http://www.kandh.com.tw/products_2.php?prod $=174 \&$ gid $=4$. 
[11] Лавров А.А., Лупин А.В. Модели в разработке систем удаленного управления (Models are in development of the systems of remote control). Proceedings of IX-th International conference "Mathematical modeling in education, science and manufacture 2015", "Pridnestrovsky University", 2015, pp. 76-77. (In Russian).

[12] Neoway M660 [online] [15.03.2018]. Available at:

$\mathrm{http}: / /$ wless.ru/producers/?action $=$ details \&id $=522 \& \mathrm{pf}=$ prod\&pf $\_$id $=35 \&$ prod $=35 \&$ tech $=2$ \& type $=34$.

[13]NI LabVIEW 2012-2016 [online] [15.03.2018]. Available at: http://www.ni.com/ru$\mathrm{ru} / \mathrm{shop} / \mathrm{labview} \cdot \mathrm{html}$.

[14]Neo_M660 GPRS Module AT command Set [online] [15.03.2018]. Available at: http://www.wless.ru/files/GSM/Neoway/Neoway_M660_AT_Command_Set_V3_9.pdf

[15]Блюм П. LabVIEW: Стиль программирования (LabVIEW: programming Style). M: "DMK Press", 2008, 400 p. (In Russian). 